\title{
Quels sont les principaux changements qui ont modifié la fonction de direction ou de direction adjointe d'établissement scolaire depuis l'avènement de la réforme de l'administration publique au Québec? What major changes have altered the profession of principal/vice- principal since the advent of Québec's Public Administration Reform? ¿Cuáles son los cambios principales que han modificado la profesión de dirección/dirección adjunta de los establecimientos escolares desde la aparición de la Reforma de la Administración Pública en Quebec?
}

\author{
France Gravelle
}

Volume 40, numéro 1, printemps 2012

La gestion et l'appropriation du changement en éducation

URI : https://id.erudit.org/iderudit/1010147ar

DOI : https://doi.org/10.7202/1010147ar

Aller au sommaire du numéro

Éditeur(s)

Association canadienne d'éducation de langue française

ISSN

0849-1089 (imprimé)

1916-8659 (numérique)

Découvrir la revue

Citer cet article

Gravelle, F. (2012). Quels sont les principaux changements qui ont modifié la fonction de direction ou de direction adjointe d'établissement scolaire depuis l'avènement de la réforme de l'administration publique au Québec? Éducation et francophonie, 40(1), 76-93. https://doi.org/10.7202/1010147ar
Résumé de l'article

Au fil des années, la réforme de l'administration publique a fait place à plusieurs changements au sein des commissions scolaires du Québec qui ont contribué à la complexification de la profession des dirigeants d'établissements scolaires, en les obligeant à être toujours prêts à rendre des comptes (reddition de comptes). La direction ou direction adjointe d'établissement occupe une position plus exigeante qu'auparavant, parce qu'elle doit maintenant faire preuve de beaucoup plus d'habiletés politiques en s'assurant de la cohérence de ses interventions au regard des encadrements nationaux de la formation des élèves québécois, de la convention de partenariat, du plan stratégique, des politiques et des normes en vigueur dans sa commission scolaire, ainsi que du plan de réussite, de la convention de gestion et du projet éducatif de son établissement. En réalité, ces changements ont eu un impact direct sur le travail prescrit et le travail réel des directions et directions adjointes d'établissements des commissions scolaires du Québec. Dans cet article, nous préciserons les distinctions entre le travail réel et le travail prescrit des directions adjointes d'établissements et nous identifierons comment cela a joué sur le projet éducatif de l'école.
Tous droits réservés (C) Association canadienne d'éducation de langue française, 2012
Cedocument est protége par la loi sur le droit d'auteur. L'utilisation des services d’Érudit (y compris la reproduction) est assujettie à sa politique d'utilisation que vous pouvez consulter en ligne. 


\section{Quels sont les principaux changements qui ont modifié la fonction de direction ou de direction adjointe d'établissement scolaire depuis l'avènement de la réforme de l'administration publique au Québec?}

France GRAVELLE

Québec, Canada

\section{RÉSUMÉ}

Au fil des années, la réforme de l'administration publique a fait place à plusieurs changements au sein des commissions scolaires du Québec qui ont contribué à la complexification de la profession des dirigeants d'établissements scolaires, en les obligeant à être toujours prêts à rendre des comptes (reddition de comptes). La direction ou direction adjointe d'établissement occupe une position plus exigeante qu'auparavant, parce qu'elle doit maintenant faire preuve de beaucoup plus d'habiletés politiques en s'assurant de la cohérence de ses interventions au regard des encadrements nationaux de la formation des élèves québécois, de la convention de partenariat, du plan stratégique, des politiques et des normes en vigueur dans sa 
Quels sont les principaux changements qui ont modifié la fonction de direction ou de direction adjointe

d'établissement scolaire depuis l'avènement de la réforme de l'administration publique au Québec?

commission scolaire, ainsi que du plan de réussite, de la convention de gestion et du projet éducatif de son établissement. En réalité, ces changements ont eu un impact direct sur le travail prescrit et le travail réel des directions et directions adjointes d'établissements des commissions scolaires du Québec. Dans cet article, nous préciserons les distinctions entre le travail réel et le travail prescrit des directions adjointes d'établissements et nous identifierons comment cela a joué sur le projet éducatif de l'école.

\section{ABSTRACT}

What major changes have altered the profession of principal/viceprincipal since the advent of Québec's Public Administration Reform?

France GRAVELLE

Québec, Canada

Over the years, the Public Administration Reform has paved the way for many changes in Québec school boards, which contributed to complexifying the profession of school administrators, requiring them to be accountable at all times. The job of principal/vice principal is now more demanding than before, since administrators now need many more political skills to ensure that their actions are consistent with national frameworks for educating Québec students, the partnership agreement, the strategic plan, policies and standards in effect in the school board, as well as the school's success plan, management agreement and educational project. In reality, these changes have had a direct impact on the actual and prescribed work involved for principals/vice principals working in Québec school boards.

\section{RESUMEN}

\section{¿Cuáles son los cambios principales que han modificado la profesión de dirección/dirección adjunta de los establecimientos escolares desde la aparición de la Reforma de la Administración Pública en Quebec?}

France GRAVELLE

Québec, Canada

A lo largo de los años, la Reforma de la Administración Pública ha dado lugar a varios cambios dentro de las comisiones escolares de Quebec, cambios que han contribuido a la complexificación de la profesión de los dirigentes de establecimientos escolares, obligándolos a estar constantemente listos para proporcionar informaciones (rendir cuentas). La dirección/dirección adjunta del establecimiento ocupa actualmente una posición mucho más exigente que anteriormente ya que deben 
Quels sont les principaux changements qui ont modifié la fonction de direction ou de direction adjointe

d'établissement scolaire depuis l'avènement de la réforme de l'administration publique au Québec?

desplegar una amplia gama de habilidades políticas, además de garantizar la coherencia de sus intervenciones con respecto a las directivas nacionales para la formación de los alumnos quebequenses, a la convención de partenariado, al plan estratégico, a las políticas y a las normas vigentes en su comisión escolar, así como con el plan para el éxito, con la convención de gestión y con el proyecto educativo de su establecimiento. En realidad, dichos cambios han tenido un impacto directo sobre el trabajo prescrito y el trabajo real de las direcciones/direcciones adjuntas de los establecimientos de las comisiones escolares de Quebec.

\section{Introduction}

L'expérience québécoise en matière de réforme de l'administration publique, amorcée en 2000 par les modifications à la Loi sur l'administration publique, visait à accroître la transparence, la responsabilisation ainsi que la reddition de comptes dans la gestion du secteur public. Tout en privilégiant les initiatives organisationnelles associées au mouvement de décentralisation ainsi que la qualité et la proximité des services offerts, cette réforme misait également sur l'atteinte de résultats mesurables. Elle encourageait l'élaboration d'une planification stratégique, d'un plan de réussite annuel ainsi que la publication d'un rapport annuel de gestion (MELS, 2008).

La décentralisation mise en œuvre en 1998 donne maintenant à l'établissement plus d'autonomie qu'auparavant, mais celle-ci se révèle fortement encadrée et contrôlée. En effet, le fonctionnement de l'établissement et l'exercice de la fonction de direction ou direction adjointe demeurent largement normalisés ou déterminés par des encadrements nationaux et régionaux de toutes natures, à savoir lois, politiques et règlements (Brassard, 2004).

De plus, les structures de travail ont évolué, les modes de vie des personnes se sont transformés, les valeurs et les habitudes ont changé dans un contexte pluraliste, tout en faisant place à une expression des droits plus marquée. Les savoirs se sont développés rapidement et circulent plus aisément, compte tenu des technologies de l'information. Mais, en même temps, les situations sont devenues plus complexes et les problèmes se posent maintenant avec plus d'acuité. L'institution scolaire est concernée au plus haut point par tous ces changements, c'est même en grande partie sur elle que la société compte pour préparer les individus à relever les défis que posent ces nouvelles situations. La formation des individus est maintenant mise au premier rang des priorités sociales par la plupart des leaders économiques et sociaux. On en fait même la clé du développement de la société de demain. Afin de se réaliser, les contenus éducatifs ont dû être adaptés, les modes de distribution des services et de collaboration ont dû également être repensés à la lumière des nouvelles réalités des populations desservies. Plusieurs façons de faire qui ont réussi par le passé ont dû 
Quels sont les principaux changements qui ont modifié la fonction de direction ou de direction adjointe

d'établissement scolaire depuis l'avènement de la réforme de l'administration publique au Québec?

être abandonnées au profit d'approches mieux adaptées (Conseil supérieur de l'éducation, 1992).

Les changements profonds auxquels on assiste dans le monde de l'éducation sont donc tributaires des modifications du contexte social en général. Délaissant un système centralisé et hiérarchisé qui préconise une pédagogie magistrale, les écoles héritent maintenant d'un pouvoir décentralisé qui favorise une pédagogie par projets inscrite dans un partenariat famille-école-communauté. Il est clair que le partenariat est lié aux grands enjeux auxquels la société doit faire face pour organiser l'existence de ses membres. Il fait partie des moyens que l'on a imaginés pour traiter des problèmes de la vie sociale (MEQ, 1997).

La structure organisationnelle de l'éducation, tout en ayant l'avantage d'être définie par des lois, est maintenant multipartite: conseil des commissaires, conseil d'établissement, direction générale de la commission scolaire, direction d'établissement et enseignants. Les différents acteurs du monde de l'éducation québécois doivent maintenant composer avec les dispositions formelles de la loi (travail prescrit) et les limites des pouvoirs sous-jacents, la compréhension de chacun quant à son rôle et aux sources de pouvoir qu'il détient et les pratiques quotidiennes à l'intérieur de cette dynamique formelle (travail réel).

Cet article a ainsi pour but d'expliquer les principaux changements survenus depuis la réforme de l'administration publique au Québec qui ont influencé le travail prescrit et le travail réel des directions adjointes d'établissements, tout en les obligeant à redéfinir le projet éducatif de l'école (structures pédagogiques et organisationnelles), en vue d'assurer la réussite du plus grand nombre d'élèves.

\section{Cadre théorique}

Le cadre théorique de cet article repose sur la théorie selon laquelle un «écart considérable existe entre l'activité théorique supposée (Loi sur l'instruction publique (180) [travail prescrit]) et l'activité déployée par les employés (tâche réelle d'une direction ou d'une direction adjointe d'établissement - travail réel) » (Terger et Bernier, 1990), depuis l'avènement de la réforme de l'administration publique québécoise.

«Les groupes de travail vivent selon des règles non écrites, non prescrites par l'organisation, mais conçues par les acteurs, négociées entre eux et selon les cas, avec les hiérarchies» (de Terssac, 1996). Selon Amalberti et Hoc (1998), «il faut comprendre l'activité et la tâche effective comme des synonymes. L'activité se définit par les opérations manuelles et intellectuelles réellement mises en jeu à chaque instant par l'opérateur pour atteindre ses objectifs, pas seulement prescrits». En réalité, les directions et les directions adjointes d'établissements des commissions scolaires francophones du Québec ont des règles et des termes qui sont propres à leur profession. Ces règles et ces termes sont teintés par leur région, par la culture de leur commission scolaire, ainsi que par celle de leur établissement. 
Quels sont les principaux changements qui ont modifié la fonction de direction ou de direction adjointe

d'établissement scolaire depuis l'avènement de la réforme de l'administration publique au Québec?

\section{Travail prescrit versus travail réel}

Plusieurs recherches concernant l'ergonomie et la psychologie du travail ont insisté sur la distinction entre la tâche prescrite (travail prescrit) et l'activité réelle (travail réel). «La tâche est ce qui est à faire, tandis que l'activité est ce qui se fait» (Leplat et Hoc, 1983, cités par Clot, 1999).

D'après Clot (1999) :

si le travail réel n'est pas conforme au travail prescrit, c'est que la femme ou l'homme ne sont pas seulement des producteurs, mais des acteurs engagés dans plusieurs mondes et plusieurs temps vécus à la fois, mondes et temps qu'ils cherchent à rendre compatibles entre eux, dont ils espèrent surmonter les contradictions en les pliant à leur propre exigence d'unité, même s'il ne s'agit là que d'un idéal.

Selon J. Leplat (1997):

[l'agent] ne peut être conçu comme un simple système d'exécution de la tâche prescrite. Cette tâche s'inscrit pour lui dans son histoire. Il ne fait pas que réaliser la tâche prescrite, mais il vise aussi, par cette réalisation, des buts personnels. L'agent redéfinit aussi cette tâche par rapport à celle des autres et la qualité de la vie collective peut être au centre de la tâche ainsi redéfinie. L'agent réalise une autre tâche que celle qui lui était proposée: la redéfinition de la tâche n'est d'ailleurs pas seulement individuelle.

Dans le cadre de cet article, nous considérons que l'avènement de la réforme de l'administration publique québécoise ainsi que plusieurs changements sociaux ont créé un écart considérable entre les tâches de direction ou de direction adjointe prescrites par la Loi sur l'instruction publique (180) et celles réalisées quotidiennement au sein de l'établissement scolaire.

\section{Collectif de travail}

Le référentiel commun de connaissances opératives n'est qu'une composante du corps social et symbolique qui s'interpose entre les personnes dans le travail; une composante seulement du genre d'activités exigées dans la situation qui en comporte d'autres. Comme l'ont bien noté les sociologues, ce référentiel élaboré en commun établit des règles non écrites, non immuables, dans une situation communicationnelle qui suppose l'élaboration commune. Il inscrit les savoir-faire considérés dans l'histoire d'un collectif: "Les savoir-faire ne peuvent pas être isolés du contexte social de leur usage, à savoir la production de règles non écrites élaborées en commun au sein d'un collectif» (de Terssac, 1992). "Cette production de règles est une construction sociale qui est aussi le moyen de constituer le collectif en tant que tel. La règle non écrite structure les diverses sortes d'échanges dans lesquels les opérateurs se trouvent engagés.» En fait, selon de Terssac, «s'il y a une régularité dans le phénomène social que constitue la règle non écrite, s'il y a contrat implicite c'est sur la nécessité, dans le cadre de finalités productives, de trouver la stratégie 
Quels sont les principaux changements qui ont modifié la fonction de direction ou de direction adjointe

d'établissement scolaire depuis l'avènement de la réforme de l'administration publique au Québec?

collective dans laquelle chacun se retrouve à un moment donné». Nous avons donc affaire à une organisation du travail réel par les travailleurs eux-mêmes, mettant leur action en «ordre»; à une formalisation collective de l'action individuelle dont le groupe professionnel se fait le sujet. «Les groupes de travail vivent selon des règles non écrites, non prescrites par l'organisation mais conçues par les acteurs, négociées entre eux et selon les cas, avec les hiérarchies» (de Terssac, 1996). En fait, les directions et les directions adjointes d'établissements des commissions scolaires du Québec ont elles aussi des règles et des termes qui sont propres à leur profession (travail réel), tout en siégeant aussi au comité consultatif de gestion (CCG - le rôle de ce comité sera décrit plus loin dans cet article) institué (Loi sur l'instruction publique, article 183) par la commission scolaire.

\section{Culture professionnelle collective}

La culture professionnelle collective permet chaque fois une réalisation étonnante: «deux personnes qui ne se connaissaient pas sont capables immédiatement de composer une équipe opérationnelle, de se coordonner efficacement pour réaliser une mission difficile dans un contexte complexe» (Jouanneaux, 1999).

Le milieu de travail est une société organisée comme un groupement plus ou moins stable d'individus "ayant entre eux des rapports qui assignent à chacun son rôle ou sa place dans l'ensemble» (Wallon, 1971, p. 289, cité par Clot, 1999).

Les normes que lui impose son appartenance au groupe obligent le sujet au travail à régler son action et à la contrôler sur autrui comme dans un miroir, bref à s'en faire une image comme extérieure à lui-même et conforme à des exigences qui en réduisent l'absolue spontanéité et l'initiale subjectivité. Il apprend à se saisir lui-même à la fois comme sujet et comme objet, comme «Soi» et comme «Lui». Il prend conscience de sa personne (Wallon, 1971, p. 295, cité par Clot, 1999).

L'analyse des situations de travail dans les activités dites «d'exécution» a donné l'occasion à l'ergonomie dans sa tradition francophone de démontrer l'écart important entre la tâche, telle qu'elle est prescrite (travail prescrit), et l'activité de ceux qui la réalisent (travail réel) (Teiger et Bernier, 1990).

\section{Travail collectif prescrit}

Le travail collectif prescrit existe parce que la tâche impose un certain nombre d'activités partagées dans un espace commun. Le collectif de travail se développe plus ou moins dans l'activité partagée d'élaboration et de pondération des règles, de la confiance et de la reconnaissance des compétences respectives entre salariés (manières de faire et manières de dire les choses qui sont relativement stabilisées dans un milieu donné). Les collectifs professionnels réalisent un travail de reconception en situation, une série de ressources partagées relie fortement ceux qui travaillent ensemble à la même tâche, comme une gamme sédimentée de techniques intel- 
Quels sont les principaux changements qui ont modifié la fonction de direction ou de direction adjointe

d'établissement scolaire depuis l'avènement de la réforme de l'administration publique au Québec?

lectuelles et corporelles tramée dans des gestes et dans des mots, des intonations. Le genre professionnel désigne un canevas de «prêts à agir» qui fait justement défaut au nouveau venu dans un milieu de travail, même s'il a appris à la perfection les modes opératoires et les techniques en formation. Le genre professionnel relève d'une histoire transpersonnelle, au sens où elle "traverse» ceux qui en ont l'expérience, sans appartenir à chacun en propre (Prot, 2007).

\section{Travail prescrit}

L'existence d'un travail de prescription dans le milieu scolaire (travail prescrit) (voir le tableau 1 = composantes et organismes exerçant des pouvoirs prescrits et/ou d'influence) est là pour témoigner qu'entre concepteurs et opérateurs, c'est bien du travail qui s'échange, au travers des subordinations sociales. Pour bien comprendre la distinction entre le travail prescrit et le travail réel, prenons comme exemple l'article 212 de la Loi sur l'instruction publique qui oblige le conseil des commissaires d'une commission scolaire à adopter une politique de maintien ou de fermeture d'école, afin que la commission scolaire puisse s'appuyer sur un document légal lorsqu'il y a une baisse significative du nombre d'élèves fréquentant une école et qu'une question de fermeture est à légitimer.

\section{Travail réel}

Le réel de la tâche des directions/directions adjointes d'établissements (travail réel) (voir le tableau 2 = composantes et organismes exerçant des pouvoirs prescrits et/ou d'influence) est donc la modification de ces conditions par le sujet et non pas ces conditions elles-mêmes. Ce n'est pas un environnement, mais un milieu d'action délimité par les initiatives que le sujet prend seul ou avec d'autres, dans des conditions qui ne sont pas pour autant fixées par lui. Le réel c'est l'action de réalisation jamais totalement prévisible - qui met le sujet aux prises avec les réalités objectives du monde, des choses et des hommes, occasions et obstacles à son développement. À titre d'exemple, une commission scolaire décide de fermer une école parce que cette dernière ne compte plus un nombre d'élèves suffisant pour être capable de payer les coûts engendrés par ses opérations. De son côté, la communauté, par l'intermédiaire du conseil d'établissement de cette école, s'oppose à cette fermeture (article 212 - Adopter une politique de maintien ou de fermeture d'école [travail prescrit]). Cette situation a automatiquement un impact sur le travail réel de la direction et/ou direction adjointe d'établissement parce que, selon la Loi sur l'instruction publique (180), la direction d'établissement a la fonction d'assister le conseil d'éta blissement dans l'exercice de ses fonctions et pouvoirs, tout en exerçant ses responsabilités sous l'autorité du directeur général de la commission scolaire (article 44) qui, lui, dépend du conseil des commissaires (articles 201 et 202). N'est-ce pas le conseil des commissaires qui adopte la politique de maintien ou de fermeture d'école au 
sein des commissions scolaires du Québec? Bref, ce genre de situation mettra inévitablement la direction d'établissement dans la position inconfortable d'exercer ses fonctions réelles en sachant très bien que cela pourrait aller à l'encontre du travail prescrit au sens de la loi.

Tableau 1. Travail prescrit des directions/directions adjointes d'établissement des commissions scolaires francophones du Québec - Loi sur I'instruction publique (180) - Composantes et organismes exerçant des pouvoirs prescrits et/ou d'influence

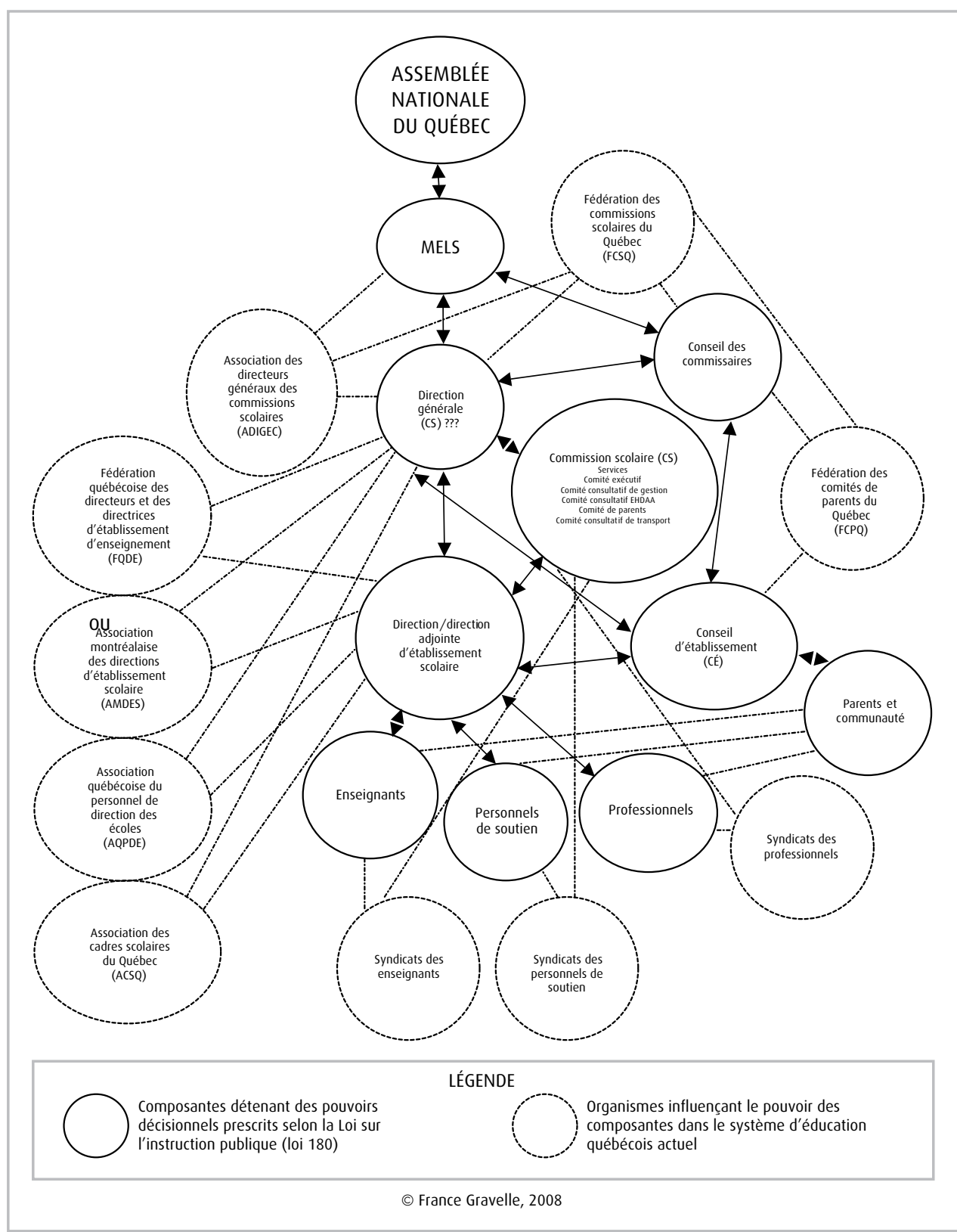


Tableau 2. Travail réel des directions/directions adjointes d'établissement des commissions scolaires francophones du Québec - Loi sur l'instruction publique (180) - Composantes et organismes exerçant des pouvoirs prescrits et/ou d'influence

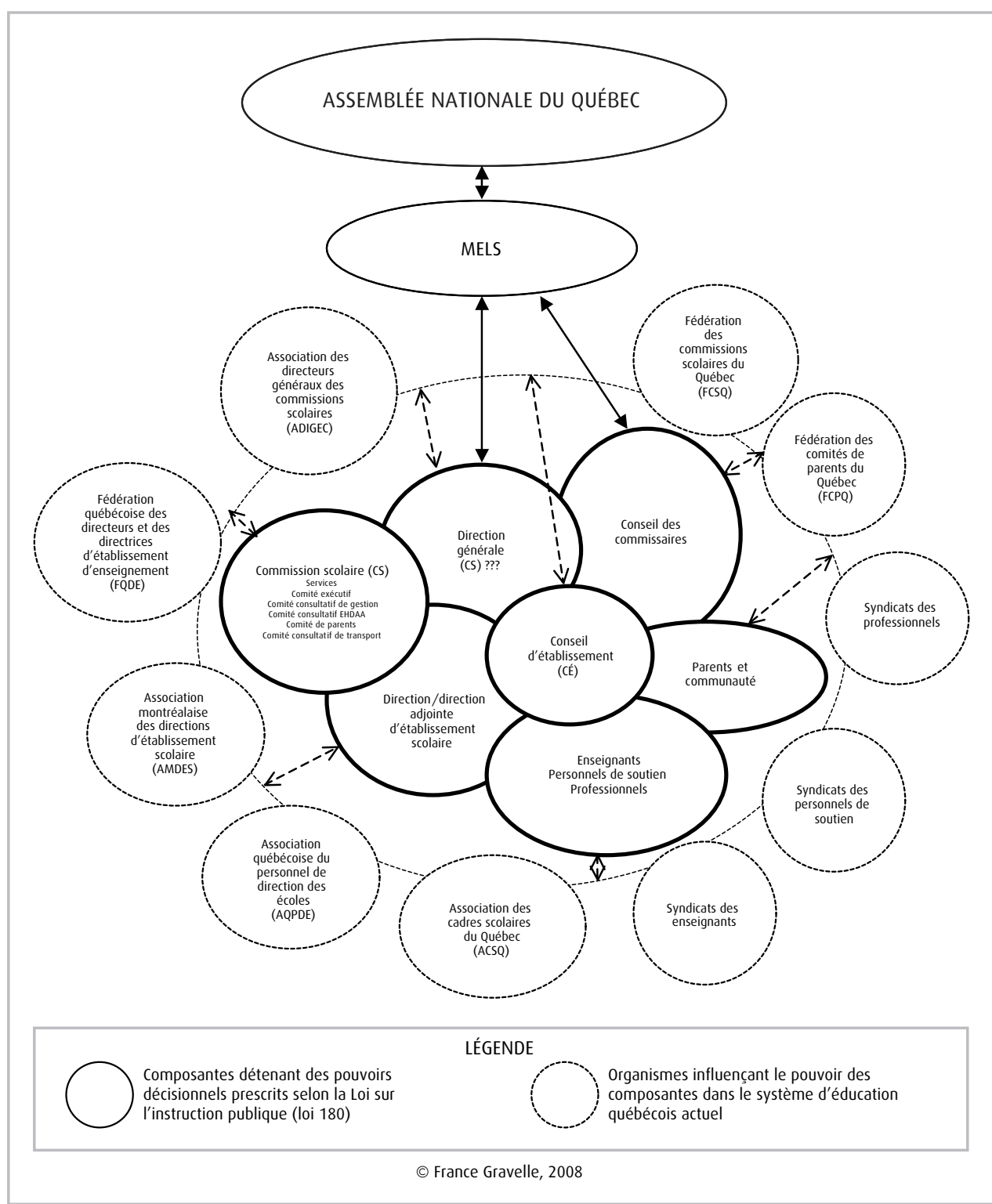


Quels sont les principaux changements qui ont modifié la fonction de direction ou de direction adjointe

d'établissement scolaire depuis l'avènement de la réforme de l'administration publique au Québec?

\section{Travail réel propre aux directions et directions adjointes d'établissements des commissions scolaires francophones du Québec}

La démocratie scolaire fait référence aux droits des citoyens de choisir, par des élections, les dirigeants des commissions scolaires qui les représenteront. Ce processus démocratique permet de confier les fonctions et les pouvoirs des commissions scolaires aux personnes qui ont obtenu la majorité des voix. La démocratie scolaire renvoie ensuite aux instances de participation, où des parents, des élèves, des enseignants, d'autres membres du personnel des établissements scolaires et des membres de la communauté sont élus par leurs pairs pour les représenter (FQDE, 2006).

Également, la commission scolaire a l'obligation d'instituer un comité consultatif de gestion (CCG) où les directions d'école sont en majorité, car l'article 183 de la Loi sur l'instruction publique stipule que:

Pour l'application des articles 96.25 et 110.13, la commission scolaire doit instituer, sous la direction du directeur général, un comité consultatif de gestion (collectif de travail) au sein duquel siègent les directeurs d'école, les directeurs de centre de formation professionnelle, les directeurs de centre d'éducation des adultes et des membres du personnel-cadre de la commission scolaire (MELS, 1998).

Si nous regardons de plus près ce collectif de travail, nous comprendrons que le comité consultatif de gestion (prescrit selon l'article 96.25) stipule que les directions d'établissement participent à l'élaboration de la planification stratégique, des politiques et des règlements de la commission scolaire. En fait, c'est un lieu de réflexion, d'échanges de vues et de partage concernant les principaux sujets qui vont influencer les décisions de gestion de la commission scolaire et des établissements, et non pas un lieu de décision. Plus précisément, cela signifie que le collectif de travail n'est pas une instance décisionnelle et qu'en plus, il se peut que ses membres puissent se faire influencer par différents paramètres légaux, sociaux et culturels provenant autant du milieu interne qu'externe de la commission scolaire ou de l'établissement.

\section{Méthodologie}

Afin de bien comprendre les paramètres pouvant influencer le travail réel d'un comité consultatif de gestion, l'approche qualitative/interprétative a été utilisée dans le cadre de la réalisation de la recherche "Analyse de l'épuisement professionnel chez les directions d'établissement des commissions scolaires francophones du Québec» (Gravelle, 1999). Elle a combiné une approche quantitative (administration de questionnaires) avec une approche qualitative (entrevues semi-directives) pour recueillir des informations auprès des directions et des directions adjointes d'établissement ayant vécu un épuisement professionnel et/ou un problème de santé relié au stress. 
Quels sont les principaux changements qui ont modifié la fonction de direction ou de direction adjointe

d'établissement scolaire depuis l'avènement de la réforme de l'administration publique au Québec?

Le type de méthodologie de cette recherche faisait place au jumelage de données quantitatives et de données qualitatives afin d'enrichir davantage les résultats escomptés. Plus précisément, une démarche qualitative/interprétative se situe au cœur même de la vie quotidienne et cherche à mieux la comprendre pour ensuite agir sur elle.

Le deuxième facteur qui légitime la pertinence de la recherche qualitative/interprétative en éducation repose sur le fait que celle-ci tient compte des interactions que les individus établissent entre eux et avec leur environnement. En réalité, selon Lorraine Savoie-Zajc (2004):

la recherche qualitative/interprétative est une forme de recherche qui exprime des positions particulières, ontologiques (sa vision de la réalité) et épistémologiques (associées aux conditions de production du savoir), dans la mesure où le sens attribué à la réalité est vu comme étant construit entre le chercheur, les participants à l'étude et même les utilisateurs des résultats de la recherche. Ces derniers, en prenant connaissance des résultats de la recherche, se mettent en position de délibération, de critique et questionnent son applicabilité et sa transférabilité dans leur propre contexte. Dans cette démarche, le chercheur et les participants à la recherche ne sont pas neutres : leurs schèmes personnels et théoriques, leurs valeurs influencent leur conduite et le chercheur tente de produire un savoir objectivé, c'est-àdire validé par les participants à la recherche. Le savoir produit est aussi vu comme dynamique et temporaire, dans la mesure où il continue d'évoluer. Le savoir est également contextuel, car les milieux de vie des participants colorent, orientent les résultats.

En somme, les directions et les directions adjointes d'établissement qui ont participé aux entrevues semi-dirigées de la recherche ont pu y décrire des situations professionnelles vécues en lien avec des paramètres pouvant influencer le comité consultatif de gestion (collectif de travail) qui, selon elles, ont favorisé l'émergence de leur épuisement professionnel.

Bref, la recherche qualitative/interprétative est source et occasion d'apprentissage, tant pour les participants que pour le chercheur. On apprend au sujet de l'envergure du discours sur le phénomène étudié (authenticité ontologique), on prend conscience de sa propre position et on peut la comparer avec celles des autres personnes (authenticité éducative).

\section{Résultats de recherche}

La recherche qualitative/interprétative de Gravelle (1999), «Analyse de l'épuisement professionnel chez les directions d'établissement des commissions scolaires francophones du Québec», a permis de recenser et de décrire les principaux paramètres pouvant influencer le travail réel d'un comité consultatif de gestion. 
Tableau 3. Paramètres pouvant influencer le comité consultatif de gestion (collectif de travail) des directions/directions adjointes d'établissements des commissions scolaires francophones du Québec

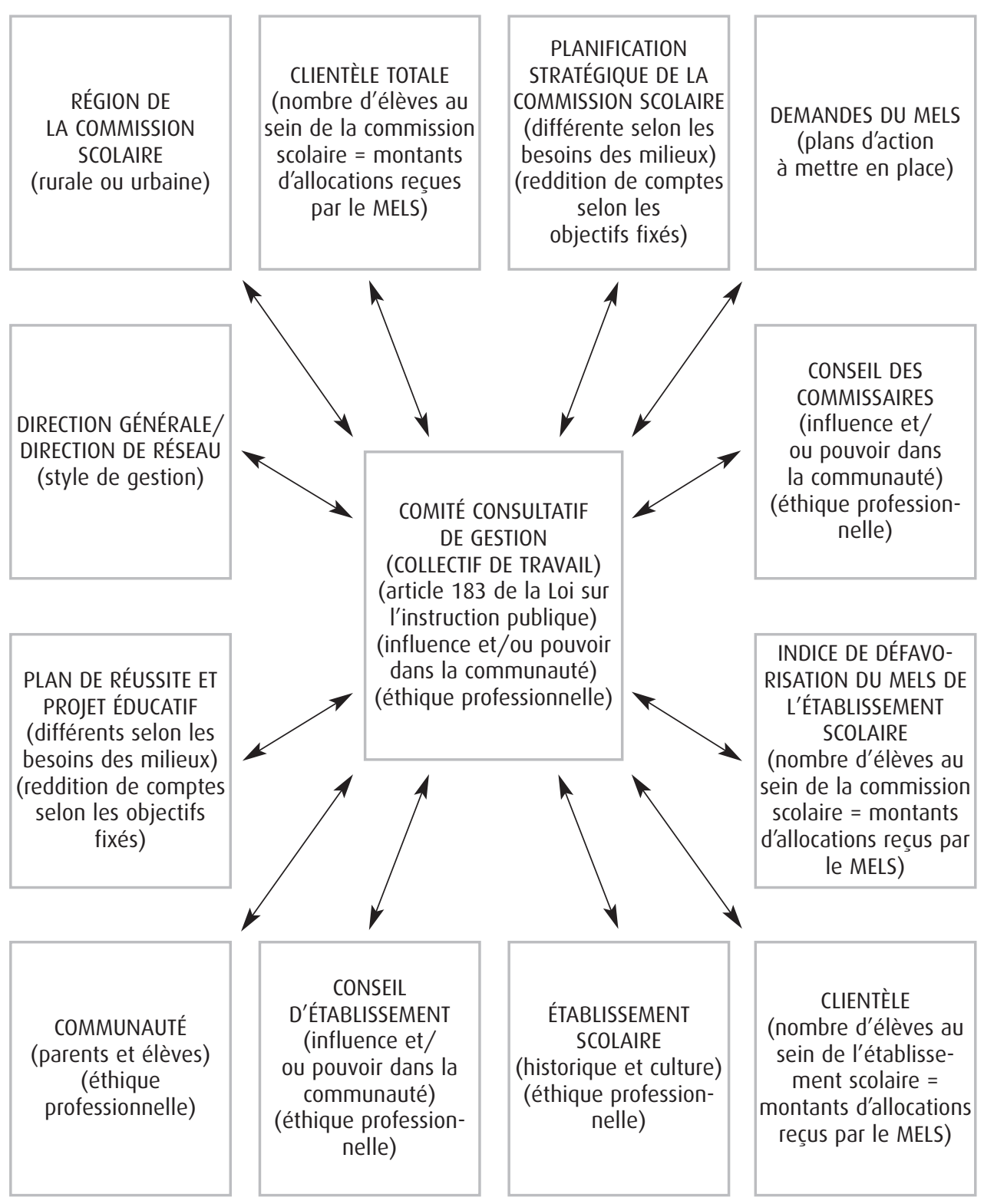

(c) France Gravelle, 2008 
Quels sont les principaux changements qui ont modifié la fonction de direction ou de direction adjointe

d'établissement scolaire depuis l'avènement de la réforme de l'administration publique au Québec?

Voici le descriptif des principaux paramètres recensés :

\section{Région de la commission scolaire}

(rurale ou urbaine)

$\mathrm{Si}$ la région est considérée comme urbaine, la commission scolaire doit de transiger avec les lois d'une ville. Par contre, si la commission scolaire se situe en zone rurale, elle fait plutôt affaire avec une MRC (municipalité régionale de comté). Dans certaines commissions scolaires, il y a des écoles en zone urbaine et d'autres en zone rurale. Ainsi, si une région est moins nantie qu'une autre, il est probable que ses besoins en ressources humaines et matérielles seront beaucoup plus grands qu'une commission scolaire ayant plus de ressources disponibles sur son territoire.

\section{Clientèle totale}

(nombre d'élèves au sein de la commission scolaire = montants d'allocations reçues par le MELS)

Tous les montants d'allocations provenant du ministère de l'Éducation, du Loisir et du Sport (MELS) tiennent compte du nombre total d'élèves inscrits au sein d'une commission scolaire. Donc, une petite commission scolaire recevra moins d'argent qu'une autre plus volumineuse en termes du nombre d'élèves au total et du nombre d'élèves considérés comme étant en difficulté d'apprentissage et/ou handicapés.

\section{Planification stratégique de la commission scolaire}

(différente selon les besoins des milieux)

(reddition de comptes selon les objectifs fixés)

Les décisions du collectif de travail doivent tenir compte des objectifs de la planification stratégique de la commission scolaire, même si certains membres des milieux (personnels - parents - membres du conseil d'établissement d'un établissement scolaire) ne sont pas d'accord avec ces dernières.

\section{Demandes du MELS}

(plans d'action à mettre en place)

Les demandes du MELS sont souvent imposées et comportent des délais très courts qui viennent parfois influencer les décisions prises par le collectif de travail. Par exemple, il arrive que le collectif de travail doive mettre en œuvre plusieurs plans d'action pendant une même année scolaire.

\section{Conseil des commissaires}

(influence et/ou pouvoir dans la communauté)

(éthique professionnelle)

L'influence et/ou le pouvoir des commissaires dans la communauté ainsi que la possibilité d'un manque d'éthique professionnelle peuvent parfois créer une certaine méfiance/tension au sein du collectif de travail. En fait, les relations qu'entretiennent certains commissaires avec des directions/directions adjointes d'établissements et/ou des membres de la communauté peuvent parfois entraver les relations de confiance entre les membres du comité consultatif de gestion. 
Quels sont les principaux changements qui ont modifié la fonction de direction ou de direction adjointe

d'établissement scolaire depuis l'avènement de la réforme de l'administration publique au Québec?

\section{Indice de défavorisation du mels de l'établissement scolaire}

(nombre d'élèves au sein de la commission scolaire = montants d'allocations reçus

par le MELS)

L'indice de défavorisation d'un établissement scolaire démontre les besoins du milieu, tant au regard des ressources que sur le plan de la réussite. Plus un indice de défavorisation est élevé, plus le milieu éprouve des besoins.

\section{Clientèle}

(nombre d'élèves au sein de l'établissement scolaire = montants d'allocations reçus par le MELS)

Les montants d'allocations provenant du MELS tiennent compte du nombre total d'élèves au sein d'une commission scolaire. À la suite de l'élaboration de politiques, ces montants sont redistribués dans les écoles selon différents modèles de distribution.

\section{Établissement scolaire}

(historique et culture)

(éthique professionnelle)

L'historique et la culture d'un établissement peuvent influencer le collectif de travail par les différentes façons de faire que l'on peut retrouver au fil des années, d'établissement scolaire en établissement scolaire et de région en région.

\section{Conseil d'établissement}

(influence et/ou pouvoir dans la communauté)

(éthique professionnelle)

L'influence et/ou le pouvoir des membres du conseil d'établissement dans la communauté ainsi que la possibilité d'un manque d'éthique professionnelle peuvent parfois créer une certaine méfiance au sein du collectif de travail.

\section{Communauté}

(parents et élèves)

(éthique professionnelle)

La possibilité d'un manque d'éthique professionnelle chez certains parents peut parfois créer une certaine méfiance au sein du collectif de travail. En fait, les relations qu'entretiennent certains commissaires avec des directions ou directions adjointes d'établissements ou des membres de la communauté peuvent parfois entraver les relations de confiance entre les membres du comité consultatif de gestion.

\section{Plan de réussite et projet éducatif}

(différents selon les besoins des milieux)

(reddition de comptes selon les objectifs fixés)

Les différences des milieux exprimées par les besoins inscrits dans les plans de réussite et dans les projets éducatifs des établissements peuvent parfois faire émerger des contradictions au sein du collectif de travail. En fait, chaque direction ou 
Quels sont les principaux changements qui ont modifié la fonction de direction ou de direction adjointe

d'établissement scolaire depuis l'avènement de la réforme de l'administration publique au Québec?

direction adjointe d'établissement a la responsabilité d'atteindre les objectifs fixés et de rendre des comptes. Pour y arriver, chaque milieu a des besoins qui peuvent être tout le contraire d'une école à l'autre.

\section{Direction générale ou direction de réseau}

(style de gestion)

Le style de gestion de la direction générale ou direction de réseau peut influencer positivement ou négativement le collectif de travail.

\section{Discussion}

La loi 180, modifiant la Loi sur l'instruction publique, est venue placer l'établissement au premier plan du réseau scolaire, au centre de l'action et de la mise en œuvre du renouveau pédagogique. Dans sa volonté de donner une plus grande autonomie sur les plans éducatif et pédagogique, le législateur a institué au cœur de chaque établissement un conseil d'établissement composé de représentants des différents groupes intéressés par l'établissement à qui il a délégué un certain nombre de fonctions et de pouvoirs. La même loi a placé à la tête de l'école une direction à qui elle a délégué la fonction d'assister le conseil d'établissement dans l'exercice de ses fonctions et pouvoirs et celle d'assurer la direction pédagogique et administrative de l'école.

En fait, aujourd'hui, les responsabilités du directeur d'établissement s'exercent sous l'autorité du directeur général de la commission scolaire (article 44) qui, lui, dépend du conseil des commissaires (articles 201 et 202) et du conseil d'établissement. Ainsi, comme l'a souligné André Brassard (2004):

l'établissement relève à la fois de l'autorité du conseil d'établissement et de l'autorité du directeur d'établissement, ce qui revient à dire que l'autorité qui s'exerce sur l'établissement est bicéphale. De même, le directeur doit assurer l'exécution des décisions du conseil d'établissement, tout en dépendant hiérarchiquement du directeur général de la commission scolaire. Il se retrouve lui aussi dans une situation de bicéphalisme.

Le devoir de rendre compte est le résultat de l'attribution de fonctions et de pouvoirs. Celui à qui la loi a délégué l'exercice d'une fonction ou d'un pouvoir doit en assumer la responsabilité, c'est-à-dire qu'il doit en rendre compte à qui de droit, selon la loi. Dit autrement, le titulaire d'une fonction ou d'un pouvoir a l'obligation de rendre des comptes à qui de droit sur la manière dont il s'en est acquitté. Rendre compte, c'est informer, expliquer et justifier l'exercice de la fonction ou du pouvoir.

Ainsi, le directeur d'établissement doit rendre compte au directeur général de la commission scolaire sous l'autorité de qui il exerce ses fonctions et pouvoirs (articles 96.8 et 96.12). De plus, la loi fait obligation au directeur d'établissement d'assister le conseil d'établissement dans l'exercice de ses fonctions et pouvoirs (article 96.13), de s'assurer de l'application des décisions du conseil d'établissement (article 96.12) et de rendre compte au conseil d'établissement de l'administration du budget de l'école (article 96.24). 
Quels sont les principaux changements qui ont modifié la fonction de direction ou de direction adjointe

d'établissement scolaire depuis l'avènement de la réforme de l'administration publique au Québec?

Depuis la décentralisation et le renouveau pédagogique, la direction ou direction adjointe d'établissement occupe maintenant une position plus exigeante. Son rôle et ses responsabilités se sont modifiés largement au cours des dernières années, appelant les personnes en fonction à s'y adapter. Bref, en plus d'élargir sa zone d'intervention sur les plans pédagogique et administratif, la direction doit aujourd'hui faire preuve de beaucoup plus d'habiletés politiques.

Comme l'a mentionné André Bellavance (2002), «la direction d'établissement doit maintenant apprendre à jongler avec les lois, les règles, les politiques, les règlements, les conventions et, surtout, avec les besoins et les attentes de ses collaborateurs et de ses clients".

Aujourd'hui, diriger en éducation nécessite beaucoup plus d'habiletés politiques qu'avant! Au cours des dernières décennies, nous sommes passés de l'exercice d'une autorité traditionnelle qui avait peu à s'expliquer à une autorité post-moderne dont la légitimité première repose sur la capacité des dirigeants à dire, à argumenter, à mobiliser, bref, à faire preuve d'un ensemble de compétences complexes associées à l'exercice d'un leadership de qualité (Pelletier, 2006).

\section{Conclusion}

Ainsi, comme on l'a expliqué précédemment, contrairement à ce qui avait cours il y a plus d'une décennie, il est maintenant important que les directions et les directions adjointes connaissent à fond chaque dossier relatif au cadre de compétences à développer, qu'elles en comprennent les incidences sur les autres en tenant compte des effets sur les personnes, qu'elles fassent participer le personnel à la prise de décision, qu'elles obtiennent l'approbation du conseil d'établissement, qu'elle puisse convaincre le commissaire, tout en acheminant l'information à la commission scolaire qui, elle, doit s'assurer de sa réalisation, en informer les parents et vaincre toutes les résistances au changement.

Bref, bien que le rôle de direction d'établissement ait grandement changé, les attentes du personnel et des parents, ainsi que les besoins des élèves, n'ont aucunement diminué. La société s'étant modifiée, elle entraîne avec elle de nouvelles obligations, de nouvelles préoccupations et de nouveaux mandats. Certaines attentes ont même augmenté, étant donné la répartition des nouveaux pouvoirs conférés aux conseils d'établissement et la responsabilité qu'a maintenant la direction d'établissement d'assurer les suivis des décisions et de préparer les travaux de cette instance. 


\section{Références bibliographiques}

AMALBERTI, R. et Hoc, J.-M. (1998). Analyse des activités cognitives en situation dynamique. Pour quels buts? Comment? Le travail humain, 61(3), 209-234.

BELLAVANCE, A. (2002). La direction d'établissement: jongleur? Le Point en administration scolaire, printemps, 6-7.

BRASSARD, A. (2001). Quelles compétences pour l'avenir? Le Point en administration scolaire, hiver, 14-15.

BRASSARD, A. (2004). La professionnalisation de la fonction de direction d'un établissement d'enseignement et le développement du champ d'études de l'administration de l'éducation. Éducation et francophonie, automne, 36-44.

CLOT, Y. (1999). La fonction psychologique du travail. Paris: Presses universitaires de France.

CLOT, Y. et FAÏTA, D. (2000). Genres et styles en analyse du travail. Concepts et méthodes. Travailler, 7-9.

CONSEIL SUPÉRIEUR DE L'ÉDUCATION (1992). Rapport annuel 1991-1992 sur l'état et les besoins de l'éducation. La gestion de l'éducation: nécessité d'un autre modèle. Québec: Gouvernement du Québec.

CONSEIL SUPÉRIEUR DE L'ÉDUCATION (2006). Rapport annuel sur l'état et les besoins de l'éducation 2005-2006 - Agir pour renforcer la démocratie scolaire. Québec: Les Publications du Québec.

FÉDÉRATION QUÉBÉCOISE DES DIRECTEURS ET DES DIRECTRICES D'ÉTABLISSEMENT D'ENSEIGNEMENT (FQDE) (2006). Les directions d'établissement - des gestionnaires d'influence et de décision. Anjou, $32 \mathrm{p}$.

FÉDÉRATION QUÉBÉCOISE DES DIRECTEURS ET DES DIRECTRICES D'ÉTABLISSEMENT D'ENSEIGNEMENT (FQDE) (2008). Les tensions de rôle chez les directrices et les directeurs adjoints d'école. Anjou.

GRAVELLE, F. (1999). Analyse de l'épuisement professionnel chez les directions d'établissement des commissions scolaires francophones du Québec. Thèse de doctorat. Université de Paris-Est.

JOUANNEAUX, M. (1999). Le pilote est toujours devant. Toulouse: Octarès.

KARSENTI, T. et SAVOIE-ZAJC, L. (2004). La recherche en éducation: étapes et approches. Sherbrooke : Éditions du CRP.

MINISTÈRE DE L'ÉDUCATION (1997). Prendre le virage du succès - Plan d'action ministériel pour la Réforme de l'éducation. Québec: Gouvernement du Québec.

MINISTÈRE DE L'ÉDUCATION (1997). Réaffirmer l'école - Rapport du groupe de travail sur la Réforme du curriculum. Québec: Gouvernement du Québec. 
Quels sont les principaux changements qui ont modifié la fonction de direction ou de direction adjointe

MINISTÈRE DE l'ÉDUCATION (2002). Loi modifiant la Loi sur le Conseil supérieur de l'Éducation et la Loi sur l'instruction publique. Québec: Gouvernement du Québec.

MINISTÈRE DE L'ÉDUCATION (2003). Les nouvelles dispositions de la Loi sur l'instruction publique. Québec: Gouvernement du Québec.

MINISTÈRE DE L'ÉDUCATION (2004). Information et reddition de compte des établissements scolaires aux parents et à la communauté. Québec: Gouvernement du Québec.

MINISTÈRE DE L'ÉDUCATION, DU LOISIR ET DU SPORT DU QUÉBEC (2006). Poursuivre le renouveau pédagogique. Québec: Gouvernement du Québec.

MINISTÈRE DE L'ÉDUCATION, DU LOISIR ET DU SPORT DU QUÉBEC (2008). Loi sur l'instruction publique. Québec: Gouvernement du Québec.

PELLETIER, G. (1998). Le leadership au sein des organisations: un regard d'Amérique. Sciences humaines, Hors série no 20, février. Aussi: Collectif (1999). Les organisations. Paris: Éditions Sciences humaines.

PELLETIER, G. (2006). Diriger au temps des réformes éducatives. Savoir, décembre, 16.

PELLETIER, G. (2007). Gouverner, c'est apprendre à ouvrir une porte sur le changement sans en forcer l'habitation. Savoir, 12(3), 10.

PELLETIER, G. (2007). La gouvernance scolaire: diriger, un métier en pleine évolution. Savoir, 12(3), 11.

PROT, B. (2004). Controverses entre les professionnels à propos de l'exercice de leur métier. Gérontologie et société, décembre, 169-181.

PROT, B. (2007). Évolutions structurelles du métier: facteurs externes ou organisationnels du vieillissement. Remettre le métier au travail: le style d'activité à l'épreuve. Dans C. Montandon et J. Trincaz (dir.), Vieillir dans le métier (p. 17-31). Paris: L'Harmattan.

TEIGER, C. et BERNIER, C. (1990). Intérêt de l'analyse ergonomique du travail pour la mise en évidence des compétences méconnues: le cas des tâches de saisie dans le tertiaire informatisé. Dans Sexe faible ou travail ardu (p. 61-70). Montréal: AFCAS.

TERSSAC de, G. (1992). L'autonomie dans le travail. Paris: Presses universitaires de France.

TERSSAC de, G. et MAGGI, B. (1996a). Le travail et l'approche ergonomique. Dans F. Deniellou (dir.), L'ergonomie en quête de ses principes (p. 77-103). Toulouse: Octarès.

TERSSAC de, G. et MAGGI, B. (1996b). Autonomie et conception. Dans G. de Terssac et E. Friedberg (dir.), Coopération et conception (p. 243-267). Toulouse: Octarès. 